\title{
Micro-patterning of Polymer-based Optical Oxygen Sensors for Lab-on-Chip Applications
}

\author{
Volker Nock* ${ }^{\mathrm{a}}$, Richard J. Blaikie ${ }^{\mathrm{a}}$, Tim David ${ }^{\mathrm{b}}$ \\ ${ }^{a}$ MacDiarmid Institute for Advanced Materials and Nanotechnology, Department of Electrical and \\ Computer Engineering, University of Canterbury, Private Bag 4800, Christchurch, NZ; \\ ${ }^{\mathrm{b}}$ Centre for Bioengineering, Department of Mechanical Engineering, University of \\ Canterbury, Private Bag 4800, Christchurch, NZ
}

\begin{abstract}
Soft-lithography and plasma etching with reactive ions were used to fabricate a polymer microfluidic cell-culture bioreactor with integrated optical oxygen sensor. Platinum(II) octaethylporphyrin ketone (PtOEPK) suspended in a microporous polystyrene (PS) matrix was spin-coated to form sensor films of variable thickness from $1.1 \mu \mathrm{m}$ to $400 \mathrm{~nm}$ on glass substrates. Sensor films were found to be smooth and well adhered. Arbitrary patterns with a minimum feature size of $25 \mu \mathrm{m}$ could be routinely replicated in the PtOEPK/PS layer using polydimethylsiloxane (PDMS) elastomer stamps as etch masks in a reactive ion etcher. No effect of plasma patterning and sensor integration by plasma bonding on the sensor signal could be observed. Detection of different gaseous and dissolved oxygen concentrations with the patterned sensor followed linear Stern-Volmer behavior. Dynamic measurement of sensor intensity as a function of different oxygen concentration showed good reproducibility and a nearly instantaneous response to gas changes. For gaseous and dissolved oxygen measurement with a patterned $400 \mathrm{~nm}$ thick film $\mathrm{I}_{0} / \mathrm{I}_{100}$ ratios of 3.2 and 2.7 were found, respectively.
\end{abstract}

Keywords: optical oxygen sensor, PtOEPK in PS, micro-patterning, RIE, sensor integration, fluorescence microscopy

\section{INTRODUCTION}

Oxygen concentration is a central parameter in biomedical engineering. Biological assay and tissue engineering applications frequently require the measurement of oxygen in solution. In cell cultures, dissolved oxygen is known to influence several important processes, such as inter-cellular signaling, growth and cell differentiation. As a key metabolite for aerobic cells, the lack of molecular oxygen in culture can ultimately lead to cell death. This makes changes in oxygen uptake of cells a powerful marker for their metabolic status, health and response to exogenous and endogenous stimuli [1]. In addition to single cell studies, oxygen consumption characteristics can further be used to determine the development status of higher organisms. This technique has the potential to provide biomedical applications and the agricultural industry with a reliable, non-intrusive tool to investigate embryogenesis, gene manipulation, cloning, assisted reproduction and transgenic animals in lab-on-chip (LOC) environments [2].

Traditional laboratory procedures for the measurement in solution require the extraction of a sample volume for external analysis. In general, this sampling approach is limited by the difficulty and time needed for extraction and analysis. With fluid volumes in the range of micro-liters and below, as found frequently in current microfluidic chips, analyte sampling constitutes a major disturbance of the system to be measured. This is a particular problem for sensing of less stable solutes such as oxygen, where retrieval is likely to significantly alter the sample characteristics [3]. Alternative in-situ sensor technologies are therefore needed for the small sample volumes found in microfluidic cell culture applications and LOC systems, and we report such an alternative here.

*volker.nock@elec.canterbury.ac.nz; phone +64 33642987 ext. 7123; fax +64 33642761 


\subsection{Oxygen Sensing}

With amperiometric electrochemical and optical sensing, two inherently different technologies currently constitute the bulk of sensors for the measurement of dissolved oxygen in biological applications. Both are tolerant to liquid exposure and exhibit the high sensitivity needed to detect the small changes of oxygen encountered in cell metabolisms. The first technology, amperiometric electrochemical oxygen sensing, is found in a variety of devices for the detection and identification of different bacteria [4] to microbial biosensors [5]. Despite continuous interest in amperiometric sensors, several significant limitations have been indentified concerning their use with biological systems and microfluidic devices. For example, organic matter has been found to decrease sensor lifetime through membrane fouling, while additional problems are posed by miniaturization itself, mainly due to the need for a reference electrode and the depletion of analyte by the sensing reaction [3].

As an alternative to the amperiometric method, optical oxygen sensors based on fluorescent dyes have recently gained increased interest for use in biomedical applications. The principle of measurement with this type of sensor relies on the quenching of either intensity or lifetime of a fluorescent dye by molecular oxygen. Fluorescence occurs upon excitation of a fluorescent molecule (fluorophore) to an exited state $S^{*}$ and subsequent relaxation back to the ground state $S_{0}$ via the emission of light [6]:

$$
\begin{gathered}
S_{0}+h v_{e x} \stackrel{\text { absorption }}{\longrightarrow} S^{*} \stackrel{\text { emission }}{\longrightarrow} S_{0}+h v_{e m} \\
S^{*}+O_{2} \stackrel{k}{\longrightarrow} \text { (quenching) }
\end{gathered}
$$

where $h$ is Planck's constant and $v_{e x}>v_{e m}$ are the frequencies of the excitation and emission light, respectively, and $k$ the molecular rate constant for oxygen quenching. Besides various other pathways, relaxation from the exited state of the fluorophore can occur via interaction with a secondary molecule. In case of oxygen, fluorescence quenching is particularly pronounced as a result of the triplet ground state of the oxygen molecule. While the majority of fluorescent dyes exhibit this characteristic quenching in the presence of oxygen, a small subgroup of dyes have been found to be especially suited due to high sensitivity and long fluorescence lifetimes. These dyes are commonly categorized into organic luminescent or organometallic compound probes and used as solution $[1,7,8]$ or immobilized on a support matrix [3,9-12] for the detection of dissolved oxygen. A concise review of probes and corresponding polymer matrices for immobilization can be found in [13].

\subsection{Sensor Patterning and Integration}

Among the selection of fluorescent dyes available, platinum(II) octaethylporphyrin ketone (PtOEPK) suspended in a microporous polystyrene (PS) matrix, has been identified as well suited for use in LOC devices due to its desirable optical properties and compatibility with standard optical components [14]. The PtOEPK dye itself exhibits both a longwave shift and an extended long-term photostability compared to other fluorescent dyes. This allows for the use of standard optical filters and makes sample handling less critical. However, while PS provides a polymer matrix with good oxygen-permeability and bio-compatibility, it is incompatible with lithographic patterning. Common organic solvents such as acetone readily dissolve PS, making it impossible to apply photoresist onto PS surfaces. Pattern lift-off in combination with pipetting of individual sensor patches has been demonstrated [9], but is potentially limited in throughput and minimum feature size due to the manual handling required.

Hence, we have developed a novel layer-by-layer fabrication process for PtOEPK/PS fluorescent sensors based on spincoating, soft lithography and reactive ion etching (RIE). A detailed description of the patterning process was previously reported in $[15,16]$. In brief, sensor layers were applied to glass substrates by spin coating. Stamps of desired sensor patterns were replicated in polydimethylsiloxane (PDMS) by soft lithography and brought into conformal contact with the PtOEPK/PS layer. Stamp patterns were transferred into the sensor layer by RIE in oxygen plasma. After etching stamps were peeled off and could be re-used. Sensor patterns were integrated with a PDMS-based microfluidic device by plasma assisted bonding and calibrated for use with gaseous and dissolved oxygen by fluorescence microscopy.

\subsection{Fluidic Chip Design}

The initial motivation for developing the sensor fabrication process stems from our previous work on the influence of microfluidic channel geometry on species transport. By applying a novel solution to the analytical model for oxygen transport and consumption by liver cells cultured in a parallel-plate system, we proposed the use of channel geometry to tailor wall shear stress and thereby the oxygen concentration profile in a bioreactor [17]. This model was successively 
used, in conjunction with computational fluid dynamic (CFD) simulations, to design the tapering of a cell-culture bioreactor geometry that is required to produce a linear shear stress gradient and a relatively constant oxygen concentration without the need for additional integrated gas supply (Fig. 1). To experimentally verify the model the oxygen sensor fabrication process was developed. Here we show the application of the fabrication process to the simultaneous fabrication of multiple sensor patterns, their integration with the microfluidic cell-culture bioreactor and characterization of dissolved oxygen measurement. The device was designed to allow for the simultaneous comparison of three different bioreactor geometries and their influence on species transport. It incorporates a standard parallel-plate bioreactor with constant shear stress, the linearly increasing shear stress bioreactor and a tapered geometry as an intermediate state.

a)

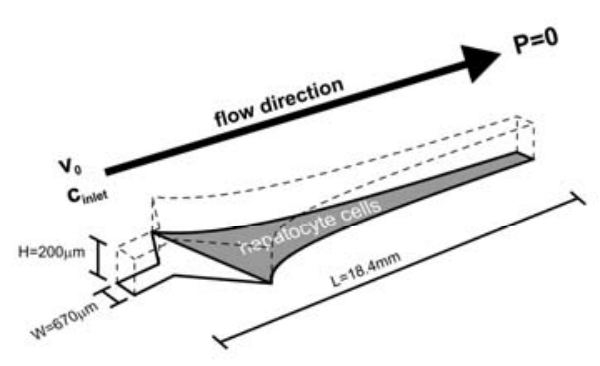

c)

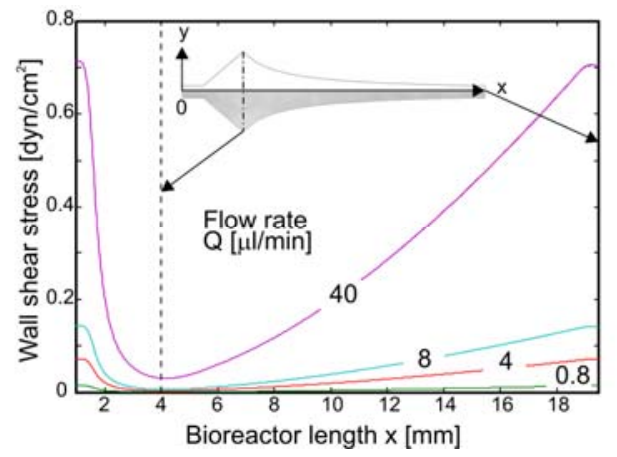

b)
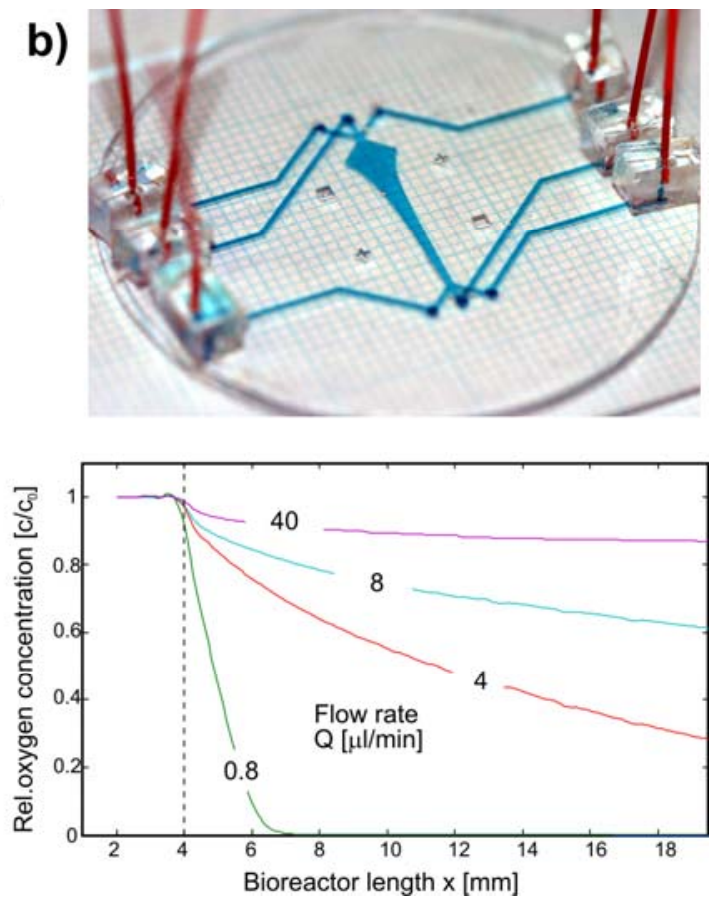

Fig. 1. Linear increasing shear-stress bioreactor: (a) Schematic of the bioreactor indicating dimensions, flow direction and simulated cell placement, with $v_{0}$ the fluid velocity, $c_{\text {inlet }}$ the oxygen concentration at the inlet and $P$ the pressure at the outlet. (b) Realization of the bioreactor in PDMS. (c) CFD simulation results for the wall shear stress (left) and relative oxygen concentration (right) along the middle of the reactor for different flow rates $Q$. Oxygen consumption of the cells was simulated to follow Michaelis-Menten kinetics.

\section{METHODS}

All the microfluidic devices presented here were fabricated by lithography in SU-8 photoresist and subsequent replica molding in PDMS. Spin-coating and soft lithography with PDMS stamps were used to pattern fluorescence-based oxygen sensors $[15,16]$. Custom patterns were transferred into the polymer sensor layer by RIE and integrated with the fluidic parts by oxygen plasma bonding. High-resolution transparencies were used as photomasks for all photolithographical steps.

\subsection{Fabrication of the Fluidic Part}

A microfluidic channel network was formed in PDMS by replica molding [18]. The master with an inverse pattern of the network was fabricated using SU-8 2075 (Microchem. Corp.) negative tone photoresist on a silicon wafer. First, SU-8 was applied by spin-coating to yield a $200 \mu \mathrm{m}$ thick film. After a $4 \mathrm{~h}$ solvent removal bake at $95^{\circ} \mathrm{C}$ on a hotplate, the resist was exposed in a Süss MA6 mask aligner. This was follow by a post exposure bake of $1 \mathrm{~h}$ at $95^{\circ} \mathrm{C}$ on a hotplate to activate resist cross-linking. Finally, development of the SU-8 was performed by immersion in (1-methoxy-2propyl)acetate and patterns were rinsed with isopropanol and blow-dried with nitrogen. To facilitate removal of the cured PDMS a fluoropolymer layer was deposited onto the resist mold. Meanwhile, liquid PDMS pre-polymer (Sylgard ${ }^{\circledR} 184$, Dow Corning) was prepared by thoroughly mixing 10:1 (w/w) base/curing agent. Prior to use the mixture was degassed 
in vacuum to remove any trapped air. The pre-polymer was then poured onto the photoresist master and cured on a hotplate at $80^{\circ} \mathrm{C}$ for $90 \mathrm{~min}$. Once cured the PDMS replica was carefully peeled off and cut to size using a scalpel before being bonded to the glass substrate with the sensor layer.

\subsection{Micro-Patterning of the Sensor Layer}

Spin coating is a commonly used process for the application of films and photoresists to flat substrates. For film formation the fluid to be coated is placed on a spinning substrate and spread by the centrifugal force to a final thickness depending on rotational speed and duration. Films applied by spin-coating exhibit high thickness uniformity and repeatability. Usually PtOEPK sensor films are fabricated by dissolving the dye in a PS/toluene solution, manually applying it to a substrate and subsequent evaporation of the solvent. In our case, we have used the dye solution on a standard spin coater to investigate the formation of PtOEPK/PS sensor films on glass substrates. The low diffusivity of oxygen, compared to PDMS and other polymers, makes glass the substrate of choice and leads to increased sensor stability and sensitivity. A 5\% w/w solution of polystyrene (average molecular weight $=200$ 000; Sigma Aldrich) in toluene was prepared and PtOEPK dye (Frontier Scientific) was added at $1 \mathrm{mg}$ per $1 \mathrm{~mL}$ of PS solution. Oxygenpermeable films of the PtOEPK/PS sensor were prepared by pipetting $200 \mu \mathrm{L}$ of the solution onto a glass microscope slide and spinning for $30 \mathrm{~s}$. Final sensor film thicknesses from $1.1 \mu \mathrm{m}$ to $400 \mathrm{~nm}$ could be produced [16]. For the purpose of determining film thickness, samples were left overnight at room temperature to evaporate the toluene and the remaining solid PtOEPK/PS film was measured using a surface profilometer. In general, the film thickness was found to decrease linearly for spin speeds from $1000 \mathrm{rpm}$ to $3000 \mathrm{rpm}$. Optical inspection of the samples further showed that spincoating produces smooth sensor films on the glass substrates. A quantitative test by blowing pressurized nitrogen onto the films demonstrated that the sensor adheres well without a substrate pre-treatment.

As described above, incompatibility of PS with common resist solvents makes it impossible to pattern the spin-coated films by applying standard photoresist. In experiments performed with conventional positive tone resist (AZ ${ }^{\circledR}$ series, Microchemicals) poor wetting of the resist on the PS film was observed [15]. Furthermore, removal of the photoresist with acetone after exposure and development, lead to the destruction of the PtOEPK/PS film surface. Hence, a different approach to sensor patterning based on polymer stamps was developed. The process mainly relies on soft polymer stamps for the definition of the pattern and oxygen plasma etching to transfer the pattern into the sensor layer.

Briefly, PDMS stamps of the sensor pattern were fabricated by a process similar to the one described above. A sensor pattern master was created in SU-8 2075 photoresist on a silicon wafer. PDMS pre-polymer was then poured onto the master, cured and peeled off. Individual stamps were cut out using a scalpel. For sensor patterning, the PDMS stamps were brought into conformal contact with the PtOEPK/PS layer. The stack was then placed in an Oxford Plasmalab ${ }^{\circledR}$ 80Plus reactive ion etcher and the layer was etched using oxygen as the reactive species. The etching conditions used were $50 \mathrm{sccm} \mathrm{O}_{2}$ flow rate, 0.55 Torr etch pressure and $200 \mathrm{~W}$ RF power applied for a typical etching time of 5 min. After plasma etching, PDMS stamps were peeled off and could be re-used. Prior to use, sensors were stored in the dark to prevent photobleaching. Partial images of the PtOEPK/PS sensor patterns were recorded using reflected light differential interference contrast (DIC) on a Nikon Eclipse 80i microscope and stitched on a PC running the Autostitch ${ }^{\mathrm{TM}}$ [19] software package.

An example of a test pattern replicated with the patterning process is shown in Fig.2. The image on the left (Fig. 2(a)) shows a SEM micrograph of the initial PDMS stamp incorporating the desired sensor shape. This stamp is inverted and placed in conformal contact with the sensor layer applied by spin-coating to a glass substrate, as described before. The stack is then put into the RIE etcher and exposed to oxygen plasma. The gas pressure during etching is chosen to be sufficiently high to allow for removal of the sensor film underneath the suspended PDMS backing of the stamp. Figure 2(b) shows an optical micrograph of the resulting pattern in a $400 \mathrm{~nm}$ thick PtOEPK/PS film after removal of the stamp. A variety of regular and irregular patterns could be replicated with a minimum feature size of $25 \mu \mathrm{m}$. The NIR emission signal of the corresponding sensor pattern after patterning is shown in Fig. 2(c). Pattern fidelity and homogeneity of the signal intensity emitted from the patterned sensor film were found to be good and at the current stage resolution is mainly limited by PDMS stamp detail. Intensity images were recorded in air before and after RIE and no effect of etching on sensor signal could be observed. The pattern resolution achieved with the low-cost process is sufficient for successful integration into common PDMS microfluidic cell-culture devices and LOCs. A further reduction in feature size should be attainable, but would require the use of a more rigid stamp material and a more complex stamp fabrication process. 

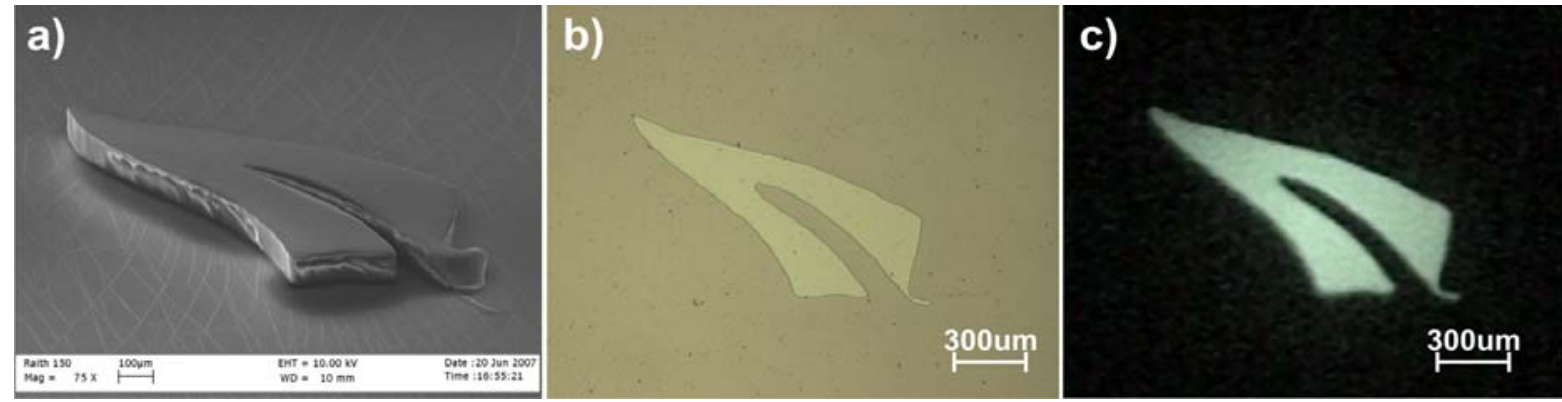

Fig. 2. Micrographs of an example pattern replicated in PtOEPK/PS sensor film. A pattern detail of the initial PDMS elastomer stamp is shown as SEM micrograph in (a) and the corresponding sensor pattern after RIE etch with the stamp as conformal mask in (b). The PtOEPK/PS film was spin-coated at $3000 \mathrm{rpm}$ to a thickness of $400 \mathrm{~nm}$. Image (c) shows the NIR sensor response of the pattern in air when illuminated with light of $595 \mathrm{~nm}$ wavelength in a fluorescence microscope. Minimum stamp detail is $25 \mu \mathrm{m}$.

\subsection{Device Integration}

Assembly of the final microfluidic device for calibration was performed by plasma bonding. Both, the surface of the PDMS channel network and the glass substrate with the sensor pattern, were activated in $\mathrm{O}_{2}$ plasma, manually aligned and brought into conformal contact. Surface activation conditions used were $50 \mathrm{sccm} \mathrm{O}_{2}$ flow rate, $75 \mathrm{mTorr}$ etch pressure and $70 \mathrm{~W}$ RF power for a duration of $10 \mathrm{~s}$ [20]. A blunt needle was used to cut access ports and external fluidic interfacing was provided by insertion of Microtight ${ }^{\circledR}$ tubing sleeves (Upchurch).

\subsection{Sensor Calibration}

Sensor calibration was performed using a Nikon Eclipse 80i fluorescence microscope in combination with a Sony Digital Handycam $^{\text {TM }}$ in nightshot mode for image acquisition. The PtOEPK dye exhibits an absorption peak at $590 \mathrm{~nm}$ and an emission peak at $760 \mathrm{~nm}$ in the near infrared (NIR), making it ideal for use with Si-based photodetectors. A special filter combination with excitation/emission filters at 595/760 nm and a $620 \mathrm{~nm}$ dichroic mirror was provided by Chroma Corp. For gaseous sensing and calibration, sensor patterns were exposed to industrial grade $\mathrm{O}_{2}$, oxygen free $\mathrm{N}_{2}$ and air. Single images were recorded after equilibration and the change of sensor intensity was analyzed using the image processing module of Matlab $^{\odot}$ (Mathworks Inc.). First, a background image with closed shutter was subtracted from each image. Then, intensity was averaged over a region of interest, and compared to a reference intensity (nitrogen saturated, $0 \% \mathrm{O}_{2}$ ). Dynamic sensor response to gaseous $\mathrm{O}_{2}$ was measured by continuously recording sensor intensity for a total duration of 20 min, while switching between applied gases. The resulting video was then digitized and the intensity change was analyzed as described above. No reduction of the sensor intensity due to photobleaching by the excitation source could be detected during this period.

For dissolved oxygen calibration, water of defined $\mathrm{O}_{2}$ concentration was flowed through the microfluidic device containing the sensor patterns. A flow-through oxygenator was built using gas-permeable silicone tubing (Silastic, Dow Corning) in a Pyrex container [21]. De-ionized (DI) water was flowed through the oxygenator and perfused with $100 \%$ instrumental grade $\mathrm{O}_{2}, 100 \%$ oxygen free $\mathrm{N}_{2}, 21 \% \mathrm{O}_{2}$ (air), a 50\% and a $80 \%$ mixture of $\mathrm{O}_{2}$ and $\mathrm{N}_{2}$. A PHD 2000 syringe pump (Harvard Apparatus) was used to provide a constant flow through the oxygenator and the microfluidic device. Dissolved oxygen levels after the oxygenator and before the device containing the PtOEPK/PS sensor pattern were monitored via a Clarke-microelectrode sensor (DO-166FT, Lazar Research). The performance of optical oxygen sensors relies on the $\mathrm{O}_{2}$ permeability of the surrounding polymer matrix. Polystyrene is the polymer of choice for PtOEPK-based sensors as the dye is readily dissolvable in the PS/toluene solution and PS forms oxygen permeable films after solvent evaporation. Furthermore, PS is considered bio-compatible and is commonly used as a substrate in cell culture applications. However, conventional photoresist solvents such as acetone attack the PS surface. This makes it difficult to structure PS with lithography. As a possible alternative to the standard lithographic process, pipetting of the sensor solution and photoresist lift-off have been proposed to fabricate PtOEPK/PS patterns [7]. This procedure required the glass substrate to be pre-etched to promote adhesion of the film. In addition, we have observed the complete sensor film lifting off after solvent evaporation and resist stripping with acetone using this procedure. To circumvent the need for manual deposition of the sensor film and to achieve more reliable patterning, we have developed a novel process based on spin-coating and plasma assisted etching of PtOEPK/PS. A detailed description of the individual process steps for film formation and patterning can be found in [16], and has been summarized briefly above. 


\section{RESULTS AND DISCUSSION}

To demonstrate applicability of the fabrication process to cell-culture devices, sensor patterns fabricated on glass microscope slides were combined with the PDMS fluidic channels by oxygen plasma bonding. An image of a fully assembled bioreactor device with external fluidic connections is shown in Fig. 3. Three cell culture chambers of different shapes and hence, different wall shear stress profiles, are integrated on the chip. The lateral tapering of the middle chamber is specifically designed to generate a linearly increasing wall shear stress along its length. Sensor patterns in the individual chambers are restricted to the indicated outlines by the plasma patterning process described before. Red and blue dye in DI water was flowed through the channels to aid visualization and to test fluidic sealing. Preliminary flow trials showed no influence of fluid shear stress on sensor layer adhesion. Sensor patterning can be tailored to provide functional areas within the microfluidic network. Figure 4 shows optical micrographs of the middle PDMS chamber with a close-up of the corresponding sensor patterns realized within the chamber. The $400 \mathrm{~nm}$ thick sensor pattern was imaged in multiple separate DIC images, which were then automatically overlaid to provide the complete micrograph. Intensity images on the left and right of Fig. 4 show the sensor response in NIR when exposed to the activation wavelength in a fluorescence microscope. Both, a large scale sensor shape inside the chamber and finer patterns in the in-/outflow channels were fabricated simultaneously with the same PDMS stamp. This will allow in situ monitoring of dissolved oxygen at various points of interest, such as the in-and outlet concentrations, as well as in the actual cell culture chamber.

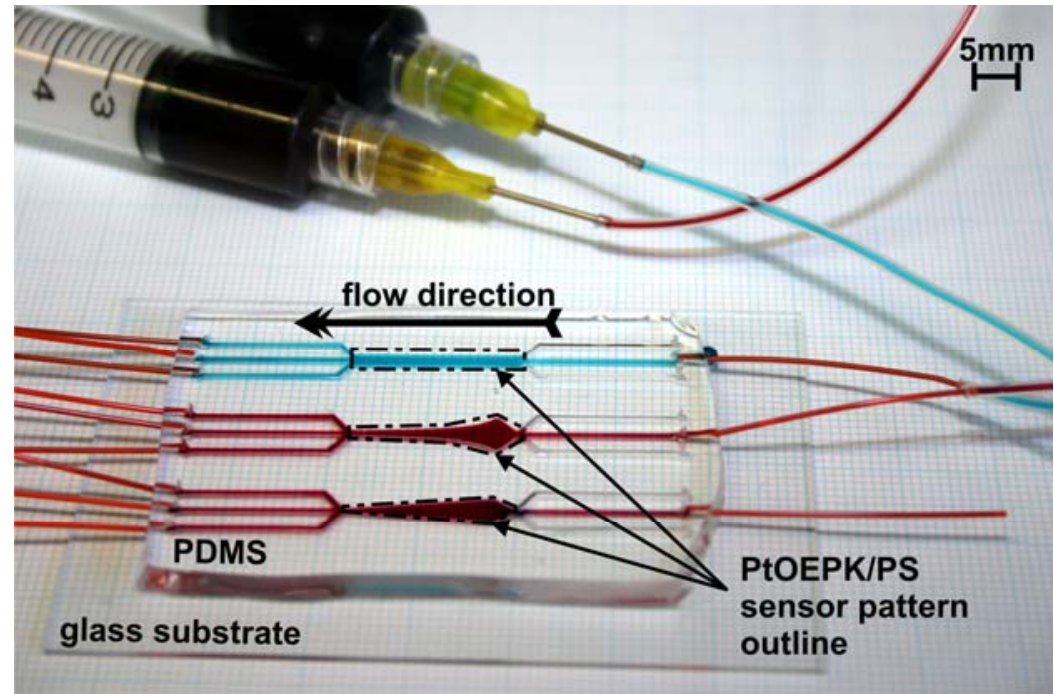

Fig. 3. Photograph of the assembled microfluidic device. Three different channel shapes with integrated oxygen sensor patterns on the underlying glass substrate are realized. Each channel shape provides a different wall shear stress profile along its length. The device is filled with dye-colored water for illustration. Calibration of the PtOEPK/PS sensor for dissolved oxygen was performed by flowing water of defined oxygen concentration through the device. Inlet oxygen concentration was measured using a flow-through Clarke electrode attached to the inlet tubing.

\subsection{Sensor Calibration}

For functional testing of the integrated oxygen sensors, a bioreactor containing the pattern depicted in Fig. 4 was fabricated. The device was placed on a fluorescence microscope and three different gaseous oxygen concentrations were flowed through the reactor. For each concentration of oxygen the static intensity response of the PtOEPK/PS layer was recorded at room temperature. Figure 5 shows a plot of 1 (fluorescent intensity) as a function of relative gaseous oxygen partial pressure. The effect of intensity quenching by molecular oxygen leads to an increase in sensor intensity from the presence of pure oxygen $\left(100 \% \mathrm{O}_{2}\right)$ to air $\left(21 \% \mathrm{O}_{2}\right)$ and to pure nitrogen $\left(0 \% \mathrm{O}_{2}\right)$, as is demonstrated by the images on the right in Fig. 5. The quenching process in presence of gaseous or dissolved oxygen for the PtOEPK/PS system and other fluorescent dyes can be described by the Stern-Volmer relationship [13]:

$$
\frac{I_{0}}{I}=1+K_{S V}^{S}\left[O_{2}\right]=1+K_{S V}^{G} p O_{2}
$$


where $K_{S V}^{S}$ and $K_{S V}^{G}$ are the Stern-Volmer constants for solution and gas, respectively, $I_{0}$ the reference intensity value in absence of oxygen, $\left[\mathrm{O}_{2}\right]$ is the oxygen concentration in solution and $\mathrm{pO}_{2}$ is the gaseous partial pressure of oxygen. The superposition of the model onto the data in graph in Fig. 5 indicates Stern-Volmer behavior. In addition to the static response, sensor dynamics for gaseous oxygen detection were investigated by alternating the flow of the respective gas over the sensor. Since intensity quenching by molecular oxygen is a reversible process, it is possible to record the sensor intensity as a function of time and oxygen concentration. Figure 6 plots the change of intensity of the PtOEPK/PS pattern for a cyclic application of air, pure oxygen and pure nitrogen gas. Sensor response was recorded under continuous microscope illumination for a duration of $20 \mathrm{~min}$. No reduction of the sensor intensity due to photobleaching was observed during measurement. The signal from the patterned PtOEPK/PS sensor film shows nearly instantaneous switching upon change of oxygen concentration, as can be seen in Fig. 6. Furthermore, good stability and reversibility under dynamic conditions can be observed for the detection of gaseous oxygen.

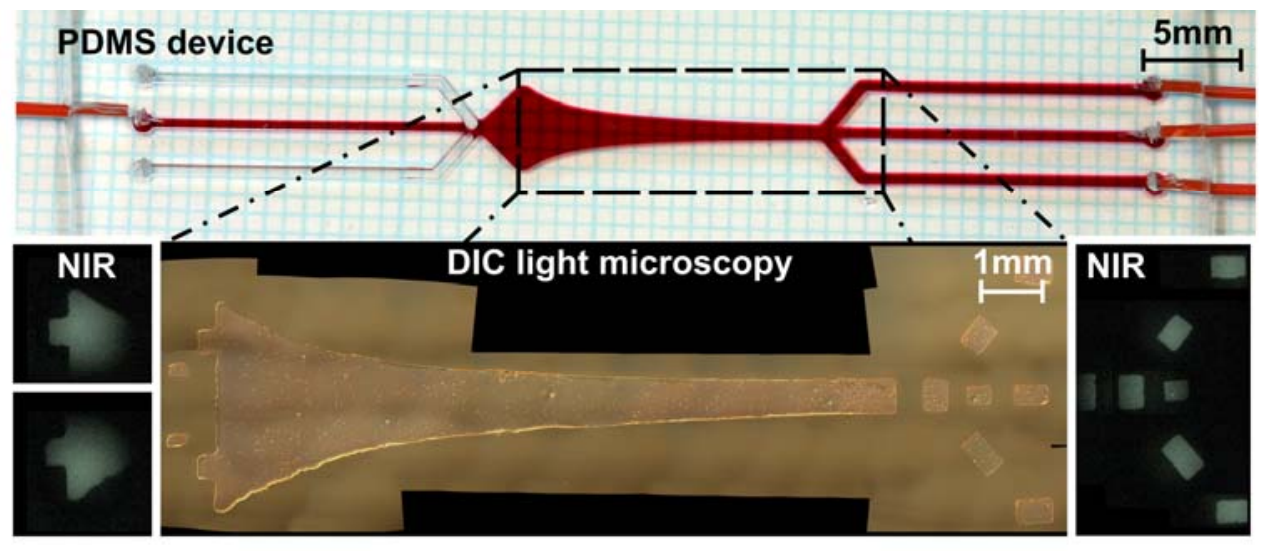

Fig. 4. Micrograph of the PDMS bioreactor device and integrated oxygen sensor pattern. The PDMS device was filled with dye-colored water for illustration purposes. Close-ups show a DIC image of the PtOEPK/PS sensor pattern defined by RIE. Pattern thickness was $400 \mathrm{~nm}$ on the glass substrate. The device was manually aligned to the sensor pattern and bonded using $\mathrm{O}_{2}$ plasma. Intensity images show sensor response of selected pattern positions in near infrared (NIR). Sensor films show good homogeneity in intensity signal. Intensity gradients in the NIR images are due to limitations of the optical system used for detection.

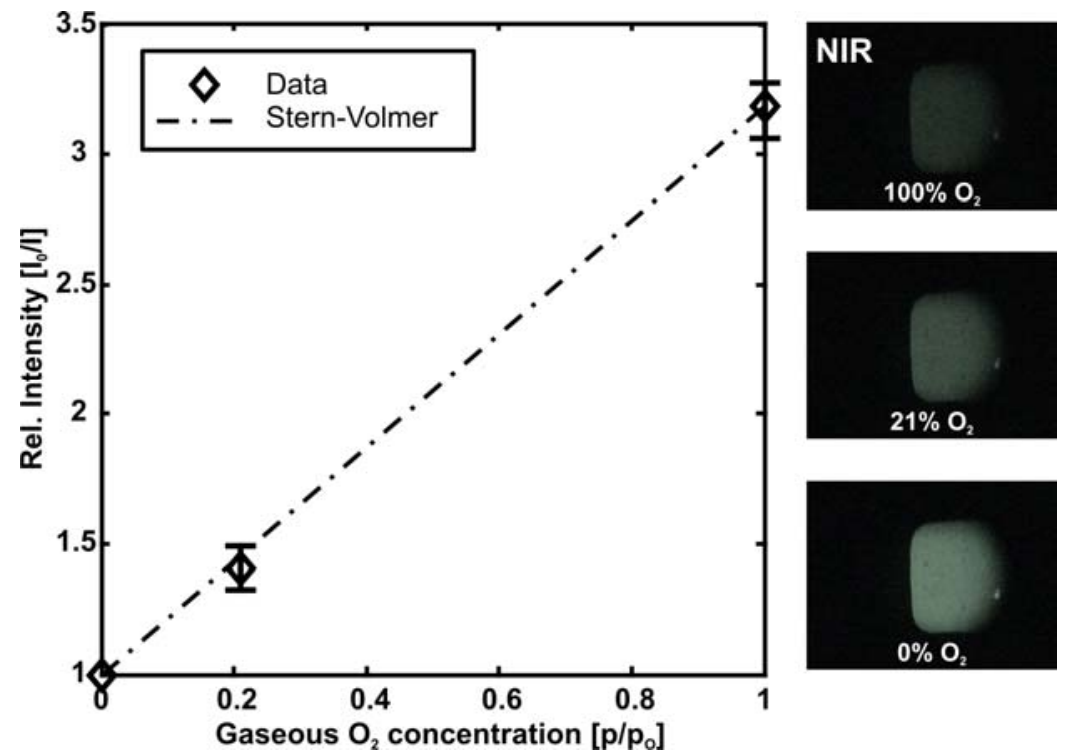

Fig. 5. Three-point calibration graph for sensor intensity as function of gaseous oxygen concentration. The dashed line corresponds to the Stern-Volmer model. Intensity images on the right show (from top to bottom) the sensor intensity change in presence of oxygen from maximum quenching at $100 \% \mathrm{O}_{2}$ to full sensor intensity at $0 \% \mathrm{O}_{2}$. 
Calibration of the sensors for dissolved oxygen was performed using the techniques described in Section 2.4. De-ionized water of five different oxygen concentrations was prepared by use of an external oxygenator. A cross-reference of the dissolved oxygen concentration at the entrance to the PDMS chip was obtained by a flow-through Clarke electrode. Intensity for a particular dissolved oxygen concentration was recorded in relation to the sensor intensity recorded for exposure to nitrogen saturated water. The relative inverse intensity versus normalized dissolved oxygen concentration for the patterned sensor layer is displayed in Fig. 7. This data was obtained for a PtOEPK/PS sensor film spin-coated at 3000 $\mathrm{rpm}$ to a thickness of $400 \mathrm{~nm}$ and intensity was measured at room temperature for a constant flow rate of $500 \mu \mathrm{L} / \mathrm{min}$. As shown in Fig. 7, the relative signal intensity from the patterned sensor is in good agreement with the Stern-Volmer model (dashed line) for dissolved oxygen.

The oxygen sensing properties of the patterned sensor are directly related to the ratio $\mathrm{I}_{0} / \mathrm{I}_{100}$, where $\mathrm{I}_{0}$ and $\mathrm{I}_{100}$ correspond to the measured intensity response from the film exposed to nitrogen and oxygen saturated conditions, respectively, and the Stern-Volmer quenching constant $K_{S V}$, found as the slope of the intensity versus oxygen concentration graph [13]. For sensor films fabricated with our process the $\mathrm{I}_{0} / \mathrm{I}_{100}$ ratio corresponds to 3.2 and 2.7 for the detection of gaseous and dissolved oxygen, respectively. These values compare favorably with previously published results for actual PtOEPK/PS sensors films deposited by pipetting [7] and other similar fluorescent dye-based sensor systems [2,3,11,12].

It can be speculated that the reduction of sensor film thickness obtained by spin-coating, compared to other application methods, increases the sensitivity of the PtOEPK/PS system due to an increase in surface-to-volume ratio of the sensor. By reducing the film thickness, the area of sensor surface in direct contact with the fluid is increased. This could have a positive impact on the dynamics of dissolved oxygen measurement by reducing the time required for diffusion from the fluid to the dye molecule in the matrix. Further investigations on the relationship between film thickness, sensitivity and dynamic behavior, as well as application of the device to cell culture, are planned.

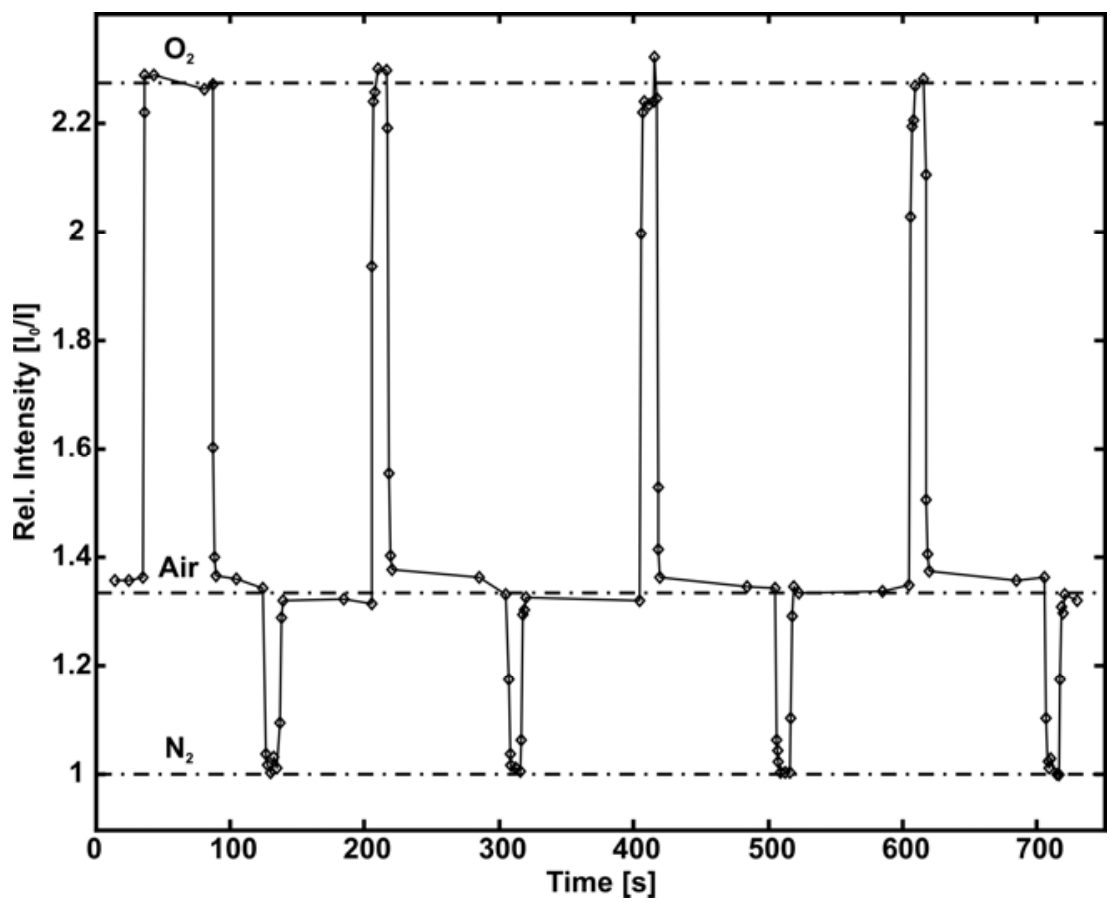

Fig. 6. Plot of the dynamic sensor response to different concentrations of gaseous oxygen. The sensor layer was exposed to $100 \% \mathrm{O}_{2}, 21 \% \mathrm{O}_{2}$ (air) and $0 \% \mathrm{O}_{2}\left(100 \% \mathrm{~N}_{2}\right)$ by flowing the respective gas through the PDMS device. Sensor intensity was continuously recorded over a duration of $20 \mathrm{~min}$ and several cycles of oxygen concentration change. No reduction of sensor signal intensity due to photobleaching was observed. The patterned PtOEPK/PS film shows fast switching response and good reproducibility. 


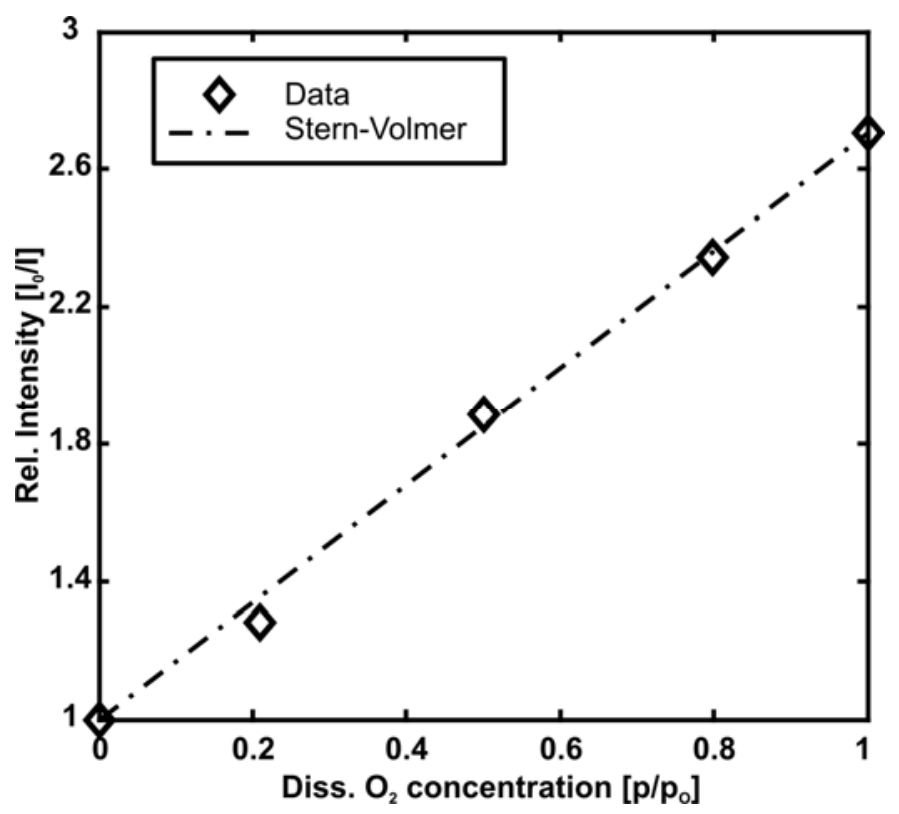

Fig. 7. Calibration plot for the measurement of dissolved oxygen concentration using the PtOEPK/PS sensor layer. Relative intensity of the sensor signal is plotted as a function of dissolved $\mathrm{O}_{2}$ concentration. Five different $\mathrm{O}_{2}$ concentrations (0 100\% $\mathrm{N}_{2}$; 0.21 Air) were prepared by flowing DI water through a silicone tubing based oxygenator and a reference Clark-electrode, $\mathrm{Q}=500 \mu \mathrm{L} / \mathrm{min}$. The dashed line corresponds to Stern-Volmer behavior and shows good agreement of the sensor response to the linear model.

\section{CONCLUSIONS}

A novel fabrication process for polymer-based oxygen sensors was used to integrate and characterize optical oxygen sensing on a microfluidic LOC device for cell-culture studies. Spin-coating as a method for the application of PtOEPK/PS sensors on glass substrates was studied and found to yield smooth, well adhered films. The final film thicknesses after solvent evaporation could be tailored from $1.1 \mu \mathrm{m}$ to $400 \mathrm{~nm}$ depending on spin-speed. Applied sensor films were further patterned by RIE with an elastomeric stamp as etch mask. Custom sensor shapes with a minimum feature size of $25 \mu \mathrm{m}$ could be reproduced and integrated with a PDMS based microfluidic channel network by PDMSglass bonding through plasma activation. Both, oxygen plasma etching and surface activation for integration were found to have no effect on the stability of the sensor signal.

The patterned sensors were characterized for exposure to different gaseous oxygen concentrations and displayed linear Stern-Volmer behavior with a typical $\mathrm{I}_{0} / \mathrm{I}_{100}$ ratio of 3.2 for a $400 \mathrm{~nm}$ thick film. Dynamic measurement of sensor intensity as a function of different oxygen concentration showed good reproducibility and nearly instantaneous response to gas changes. Application of the patterning process to dissolved oxygen sensing was demonstrated by measurement of oxygen concentration in solution inside the microfluidic device. Detection of dissolved oxygen using the PtOEPK/PS sensor patterns was found to follow the Stern-Volmer model with an $\mathrm{I}_{0} / \mathrm{I}_{100}$ ratio of 2.7.

The fabrication process provides reproducible patterning of optical oxygen sensors based on polymer encapsulated fluorescent dyes. It allows for straightforward integration of in-situ oxygen measurement in bioreactor and LOC devices. The techniques used are readily available micro-engineering processes and allow for wafer-level processing. While demonstrated here for PtOEPK/PS films, the fabrication process is not limited to this sensor system and has the potential to be applied to other polymer/dye combinations and a wide range of cell-culture and LOC applications.

\section{ACKNOWLEDGEMENTS}

The authors would like to thank Erwin Berthier for fruitful discussions and Helen Devereux and Gary Turner for technical assistance. 


\section{REFERENCES}

1 F. C. O’Mahony, C. O’Donovan, J. Hynes, T. Moore, J. Davenport and D. B. Papkovsky, "Optical Oxygen Microrespirometry as a Platform for Environmental Toxicology and Animal Model Studies", Environ. Sci. Technol. 39, 5010-5014 (2005).

2 C.-C. Wu, T. Saito, T. Yasukawa, H. Shiku, H. Abe, H. Hoshi and T. Matsue, "Microfluidic chip integrated with amperiometric detector array for in situ estimating oxygen consumption characteristics of single bovine embryos," Sens. Actuators, B, 125(2), 680-687 (2007).

3 D. A. Chang-Yen and B. K. Gale, “An integrated optical oxygen sensor fabricated using rapid-prototyping techniques”, Lab Chip 3, 297-301 (2003).

4 J. Karasinski, L. White, Y. C. Zhang, E. Wang, S. Andreescu, O. A. Sadik, B. K. Lavine and M. Vora, "Detection and identification of bacteria using antibiotic susceptibility and a multi-array electrochemical sensor with pattern recognition." Biosens. Bioelec. 22(11), 2643-2649 (2007).

5 E. Akyilmaz, A. Erdogan, R. Ozturk and I. Yasa, "Sensitive determination of L-lysine with a new amperiometric microbial biosensor based on Saccharomyces cerevisiae yeast cells," Biosens. Bioelect. 22(6), 1055-1060 (2007).

6 S. Fischkoff and J. M. Vanderkooi, "Oxygen diffusion in biological and artificial membranes determined by the fluorochrome pyrene," J. Gen. Physiol. 65(5), 663-676 (1975).

7 J. Alderman, J. Hynes, S. M. Floyd, J. Krüger, R. O’Conner and D. B. Papkovsky, "A low-volume platform for cellrespirometric screening based on quenched-luminescence oxygen sensing", Biosens. Bioelec. 19, 1529-1535 (2004).

8 D. Sud, G. Mehta, K. Mehta, J. Linderman, S. Takayama and M.-A. Mycek, "Optical imaging in microfluidic bioreactors enables oxygen monitoring for continuous cell culture," J. Biomed. Opt. 11(5), 050504-050503 (2006)

9 A. P. Vollmer, R. F. Probstein, R. Gilbert and T. Thorsen, "Development of an integrated microfluidic platform for dynamic oxygen sensing and delivery in a flowing medium", Lab Chip 5, 1059-1066 (2005).

10 P. Roy, H. Baskaran, A. W. Tilles, M. L. Yarmush and M. Toner, "Analysis of Oxygen Transport to Hepatocytes in a Flat-Plate Microchannel Bioreactor", Ann. Biomed. Eng. 29, 947-955 (2001).

11 X. Xiong, D. Xiao and M. M. F. Choi, "Dissolved oxygen sensor based on fluorescence quenching of oxygensensitive ruthenium complex immobilized on silica-Ni-P composite coating," Sens. Actuators, B, 117(1), 172-176 (2006).

12 S. Lee, B. L. Ibey, G. L. Cote and M. V. Pishko, "Measurement of pH and dissolved oxygen within cell culture media using a hydrogel microarray sensor," Sens. Actuators, B, In Press, Corrected Proof.

13 Y. Amao, "Probes and polymers for optical sensing of oxygen," Microchim. Acta 143(1), 1-12 (2003).

14 D. B. Papkovsky, G. V. Ponomarev, W. Trettnak and P. O’Leary, "Phosphorescent Complexes of Porphyrin Ketones: Optical Properties and Application to Oxygen Sensing", Anal. Chem. 67, 4112-4117 (1995).

15 V. Nock, R. J. Blaikie and T. David, "Plasma Patterning of Optical Oxygen Sensors", presented at $33^{\text {rd }}$ Conf. for Micro- \& Nano-Eng. MNE (2007).

16 V. Nock, R. J. Blaikie and T. David, "Plasma Patterning of Optical Oxygen Sensors", Microelec. Eng., submitted.

17 V. Nock, R. J. Blaikie and T. David, "Microfluidics for Bioartificial Livers", NZ Med. J. 120(1252), 2-3 (2007).

18 Y. Xia and G. M. Whitesides, "Soft Lithography," Ang. Chem. Int. Ed. 37(5), 550-575 (1998).

19 M. Brown, http://www.cs.ubc.ca/ mbrown/autostitch/autostitch.html

20 B.-H. Jo, L. M. Van Lerberghe, K. M. Motsegood and D. J. Beebe, "Three-Dimensional Micro-Channel Fabrication in Polydimethylsiloxane (PDMS) Elastomer", J. MEMS 9(1), 76-81 (2000).

21 R. L. Hamilton, M. N. Berry, M. C. Williams, E. M. Severinghaus, "A simple and inexpensive membrane "lung” for small organ perfusion", J. Lipid Res. 15, 182-186 (1974). 